Journal of Geodesy manuscript No.

(will be inserted by the editor)

\title{
High tilt susceptibility of the Scintrex CG-5 relative
}

\section{gravimeters}

\section{R Reudink · R Klees · O Francis · J Kusche ·}

R Schlesinger - A Shabanloui · N Sneeuw · L

\section{Timmen}

Received: date / Accepted: date

R. Reudink

Delft University of Technology

Tel.: +31-152785728

E-mail: r.h.c.reudink@tudelft.nl

R. Klees

Delft University of Technology

Tel.: +31-152785100

E-mail: r.klees@tudelft.nl

O. Francis

University of Luxembourg

Tel.: +352-4666446264

E-mail: olivier.francis@uni.lu

J. Kusche

University of Bonn

Tel.: +49-228732629

E-mail: jkusche@geod.uni-bonn.de

R. Schlesinger

University of Stuttgart 
Abstract We report on the susceptibility of the Scintrex CG-5 relative gravimeters to tilting, that is the tendency of the instrument of provide incorrect readings after being tilted (even by small angles) for a moderate period of time. Tilting of the instrument can occur when in transit between sites usually on the backseat of a car even using the specially designed transport case. Based on a series of experiments with different instruments, we demonstrate that the readings may be offset by tens of $\mu \mathrm{Gal}$. In addition, it may take hours before the first reliable readings can be taken, the actual time depending on how long the instrument had been tilted. This sensitivity to tilt in combination with the long time required for the instrument to provide reliable readings has not yet been reported in the literature and is not addressed adequately in the Scintrex CG-5 user manual. In particular, the inadequate instrument state cannot easily be detected by checking the readings during the observation or by reviewing the final data before leaving a site, pre-cautions suggested by Scintrex Ltd.

Tel.: +49-71168583473

E-mail: rs@gis.uni-stuttgart.de

A. Shabanloui

University of Bonn

Tel.: +49-228739360

E-mail: akbar.shabanloui@geod.uni-bonn.de

N. Sneeuw

University of Stuttgart

Tel.: +49-71168583389

E-mail: nicolaas.sneeuw@gis.uni-stuttgart.de

L. Timmen

Leibniz University Hannover

Tel.: +49-5117623398

E-mail: timmen@ife.uni-hannover.de 
In regional surveys with car transportation over periods of tens of minutes to hours, the gravity measurements can be degraded by some $10 \mu \mathrm{Gal}$. In order to obtain high-quality results in line with the $\mathrm{CG}-5$ specifications, the gravimeters must remain in upright position to within a few degrees during transits. This requirement may often be unrealistic during field observations, particularly when observing in hilly terrain or when walking with the instrument in a backpack.

Keywords Scintrex CG-5 autograv $\cdot$ hysteresis $\cdot$ tilt $\cdot$ susceptibility to tilt $\cdot$ accuracy degradation

\section{Introduction}

The CG-5 AutoGrav of Scintrex Ltd. is one of the most widely used relative gravimeters. The CG-5 is a slightly modified version of the CG-3M, which has been on the market since 1992. The instrument has been designed particularly for high-precision $(<10 \mu \mathrm{Gal}$ standard deviation) relative gravity applications (Scintrex Ltd., 2012a). Since the release of the CG-5 in 2002, it has been used to support absolute gravimetry (vertical gravity gradient determination), to observe first- and second-order gravity control networks, to monitor hydrocarbon and geothermal reservoirs, and to study tectonics and volcanology. According to Scintrex Ltd. (2012a) the standard deviation of the measurements at any site should be better than $5 \mu \mathrm{Gal}$ under typical noise conditions. This high precision has been confirmed in several studies, e.g., Flury et al. (2007), Zumberge et al. (2008), Lederer (2009), Jacob et al. (2010), Christiansen et al. (2011), Yushkin (2011), Parseliunas et al. (2008, 2011, 2013).

In April 2012, two Scintrex CG-3M (S/N 0004495 and S/N 0004509) and one Scintrex CG-5 (S/N 050300110) relative gravimeters were used in an attempt to improve the accuracy of absolute gravity observations at five stations in the Netherlands. Ties were measured 
between the five stations using the star method (Torge 1989), a technique that allows for an precise monitoring and determination of the instrument drift. During the surveys, the instruments were transported in the special transport cases on the back seat of a four-person car with a seat-belt holding the instruments in place. Scintrex Ltd. uses the same transport set-up when calibrating the CG-5 in the field (Scintrex Ltd., 2012b). The transit time to and from the base station varied between 1.5 and 2 hours. Transit was typically characterised by travel along highways in flat terrain without exposing the instruments to strong vibrations or shocks. The instruments were handled with care when carrying them to and from the vehicle avoiding shocks and jolts. Instrumental settings were chosen and the instruments were operated according to the manual instructions (Scintrex Ltd., 2005, 2012b). After arrival at a site, we levelled the instruments and let them settle for at least 30 minutes. Then, the instruments were powered up and left to observe for about 30 minutes. The readings were corrected for tides and drift. A total of four lines were measured with each instrument down and back, providing 24 measured gravity differences. The gravity differences could also be compared with a long record of FG-5 absolute gravity measurements. From this record, the gravity difference between two stations is known with a standard deviation of about 3-4 $\mu \mathrm{Gal}$.

The gravity differences per line are summarised in Table 1 . The observations reveal systematic deviations between forward and backward measurements of tens of $\mu \mathrm{Gal}$ with a maximum value of $74.6 \mu \mathrm{Gal}$. The RMS difference is $72.0 \mu \mathrm{Gal}$ (CG-5 S/N 050300110), 7.4 $\mu \mathrm{Gal}$ (CG-3M S/N 0004495), and 31.6 $\mu \mathrm{Gal}$ (CG-3M S/N 0004509), respectively. A comparison with FG-5 data reveals also significant deviations of up to $-104.0 \mu \mathrm{Gal}$. The RMS difference computed for all forward and backward measurements is $41.0 \mu \mathrm{Gal}$ (CG-5 S/N 050300110), 26.2 $\mu$ Gal (CG-3M S/N 0004495), and 63.4 $\mu$ Gal (CG-3M S/N 0004509), respectively. 
Table 1 Results of the April 2012 campaign per line and relative gravimeter. Column (a) represents the difference between the forward and backward measurement for that instrument. Column (b) reports the difference of the forward relative gravity values with respect to observations from the FG-5 absolute gravimetry measurements. Column (c) is the same as column (b) except for the backward measurements. All units are in $\mu \mathrm{Gal}$ unless otherwise stated. The standard deviation of the gravity differences from the FG-5 absolute gravimetry observations is $3-4 \mu \mathrm{Gal}$.

\begin{tabular}{|c|c|c|c|c|c|c|c|c|c|c|}
\hline \multirow[t]{2}{*}{ line } & \multirow{2}{*}{$\begin{array}{l}\text { gravity difference (FG-5) } \\
\qquad[\mathrm{mGal}]\end{array}$} & \multicolumn{3}{|c|}{ CG-5 S/N 050300110} & \multicolumn{3}{|c|}{ CG-3M S/N 0004495} & \multicolumn{3}{|c|}{ CG-3M S/N 0004509} \\
\hline & & (a) & (b) & (c) & (a) & (b) & (c) & (a) & (b) & (c) \\
\hline 1 & -90.363 & 74.6 & -49.5 & -25.1 & -11.6 & -21.1 & 32.7 & 42.1 & 61.9 & -104.0 \\
\hline 2 & -80.574 & 71.5 & -25.4 & -46.1 & 3.5 & -18.2 & 14.7 & 30.9 & 22.2 & -53.1 \\
\hline 3 & 31.648 & 67.6 & -65.3 & -2.3 & 5.2 & -34.3 & 29.1 & 34.1 & -48.8 & 14.7 \\
\hline 4 & 127.942 & 74.4 & -54.3 & -20.1 & -6.9 & -22.8 & 29.7 & 10.0 & -81.1 & 71.1 \\
\hline \multicolumn{2}{|c|}{ RMS difference forward/backward } & \multicolumn{3}{|c|}{72.0} & \multicolumn{3}{|c|}{7.4} & \multicolumn{3}{|c|}{31.6} \\
\hline \multicolumn{2}{|c|}{ RMS difference to FG-5 } & \multicolumn{3}{|c|}{41.0} & \multicolumn{3}{|c|}{26.2} & \multicolumn{3}{|c|}{63.4} \\
\hline
\end{tabular}

Figures 1 and 2 show the data collected at the base station during the April 2012 campaign as function of time for the CG-5 S/N 050300110 and the CG-3M S/N 0004495. One day before the beginning of the campaign the instruments were moved from the campus of TU Delft to the base station. The transit took about 1:45 hours. After arrival at the base station, the instruments were levelled and powered up to record over night to the morning of the next day. Thereafter, the instruments were moved to the next site and returned to the base station the same day. After the arrival at the base station, the instruments were again levelled. After 30 minutes the instruments were powered up and data were collected for approximately 30 minutes. Thereafter, the instruments were switched off, but stayed levelled. The next day, another 30 minutes of data were collected at the base station before the instruments were moved to the next site. Figure 1 shows that the initial readings of the CG-5 S/N 50300110 are offset by at least $80 \mu \mathrm{Gal}$; thereafter the offset decays more or less a logarith- 
mically. Approximately 10 hours of operation is required before the offsets are within the instrument's noise level. Results for the CG-3M S/N 0004509 are similar, though the initial offsets do not exceed $50 \mu \mathrm{Gal}$ (not shown). In contrast to the CG-5 S/N 050300110 and CG-3M S/N 0004509 meters, the CG-3M S/N 0004495 meter does not show a significant initial offset c.q. recovery time as Fig. 2 shows.

These results indicate that the instruments are adversely affected by the transport between the sites. A reconstruction of the transport conditions revealed that the instruments were tilted by about $8^{\circ}$ over the course of transit, which took between one and two hours. These transport conditions are not unusual. Scintrex Ltd. report similar conditions when they calibrated the CG-5 meters on the Orangeville Calibration Line (Scintrex Ltd. (2012b), page 5-37). The CG-5 user manual contains a special paragraph on Transporting and Handling (Scintrex Ltd. (2012b), pages 4-2 and 4-3) and on Elastic Hysteresis Effects (Scintrex Ltd. (2012b), pages B-1 till B-3). However, this information is insufficient as it does not clearly identify the problem of prolonged tilts and the precautions the customer must take to avoid errors in the observed gravity values, which in many cases far exceed the noise level of the CG-5. Related to the transport of the CG-5 meters, Scintrex Ltd. (Scintrex Ltd. (2012b), page 4-2 and 4-3) recommends i) placing the instrument "on a compliant surface, either a padded seat or a pad made from other shock absorbing material and fasten it down with a flexible binding material" when transported; ii) allowing the instrument to settle for a few minutes after being levelled before starting a reading; iii) keeping the instrument preferably in and upright position during shipment. And further down in the text "To obtain the highest accuracy, after unavoidable rough transport, allow the Autograv to settle for a few minutes after being levelled on its tripod before starting a reading". According to Scintrex Ltd. (Scintrex Ltd. (2012b), page B-2 and B-3), elastic hysteresis effects "do not build up to any extent because the bipolar, high frequency changes in tension on the spring effectively cancel out" 


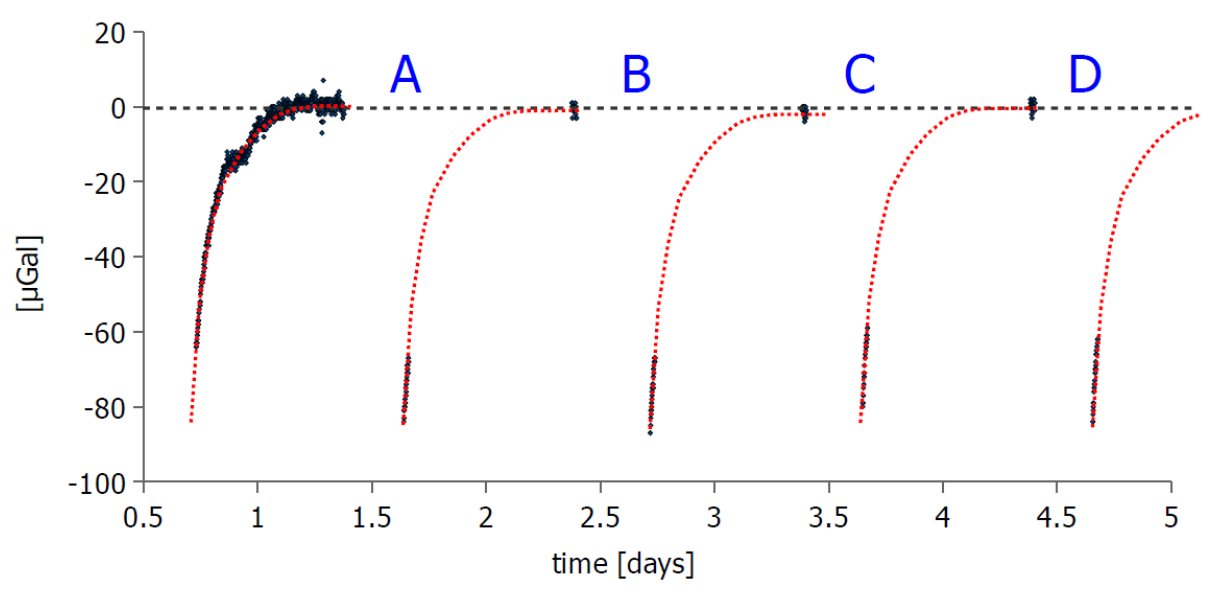

Fig. 1 Time series of tide and drift corrected readings of the CG-5 S/N 050300110 at the base station during the April 2012 campaign. Recorded readings are shown as black dots; the red curve indicates the expected behaviour of the readings. Line 1 @ day 1 (A), line 2 @ day 2 (B), line 3 @ day 3 (C), line 4 @ day 4 (D). Note the significant offset of more than $80 \mu \mathrm{Gal}$ after returning to the base station and the time before readings stabilise.

when the CG-5 is in transit. This situation relates to random movements of the beam, but not to the situation when the beam rests against one of the capacitor plates for an extended period of time.

In order to get more insight into the tilt susceptibility of the CG-5 meters, a series of tests were performed. These tests will be reported on in Section 2.

\section{Tests and results}

In this section we develop and report on a number of tests that were performed to better understand the effect of tilting on the CG-5. The tests were designed to find an answer to the following questions:

1. Do the initial offset and recovery time depend on the duration of tilt? 


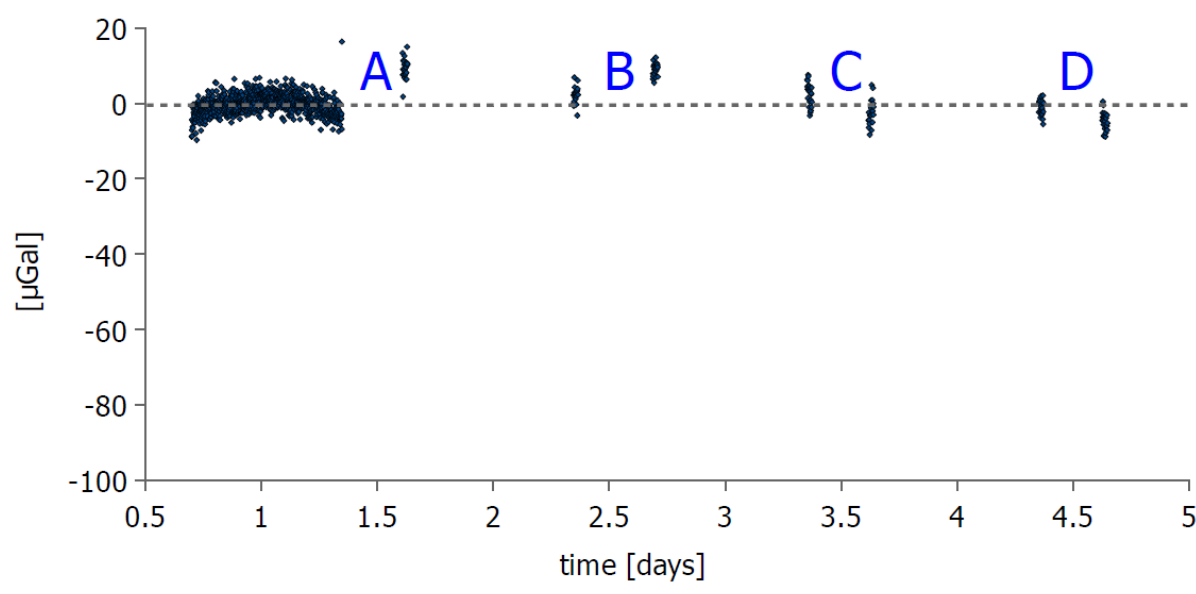

Fig. 2 Time series of tide and drift corrected readings of the CG-3M S/N 0004495 at the base station during the April 2012 campaign. Recorded readings are shown as black dots. Line 1 @ day 1 (A), line 2 @ day 2 (B), line 3 @ day 3 (C), line 4 @ day 4 (D). No significant offset is observed after return to the base station.

2. Is there an initial offset for any tilt angle or does there exist a critical tilt angle, which when not exceeded, does not cause any offset?

3. Is the susceptibility to tilt a problem of the CG-5 S/N 050300110 or is it an overall problem of the CG-5?

We focus primarily on the CG-5, because the CG-3M is no longer supported by Scintrex Ltd. However, three CG-3M meters (two from TU Delft and one from the Leibniz University Hannover) were tested, as well, because these instruments are still widely used in practice. However, we skip the details related to the CG-3M meters and only present a summary of the results at the end of this Section. In order to address the third question, the same experiments were conducted at the Universities of Luxembourg, Bonn, and Stuttgart with their Scintrex CG-5 gravimeters. Table 2 provides some information about the gravity meters involved in the experiments.

To answer the first question, i.e., Do initial offset and recovery time depend on the temporal duration of the tilt?, the following experimental set-up was chosen: the instrument 
Table 2 CG-5 and CG-3M meters used in the experiments

\begin{tabular}{llll}
\hline gravimeter & S/N & host & date of purchase \\
\hline CG-3M & 0004495 & Delft University of Technology & April 2001 \\
& 0004509 & Delft University of Technology & November 2001 \\
& 0004492 & Leibniz University Hannover & August 2001 \\
CG-5 & 050300110 & Delft University of Technology & March 2005 \\
& 110940807 & University of Bonn & September 2011 \\
& 021210010 & University of Luxembourg & January 2003 \\
& 060840191 & University of Stuttgart & August 2006 \\
\hline
\end{tabular}

is levelled, turned on, and readings are recorded for 60 minutes. Then, the instrument is turned off, tilted by $8^{\circ}$ and kept tilted for $1,3,5,8,10,13,27,50,74$, and 100 minutes, respectively. Thereafter, the instrument is levelled, turned on, and readings are recorded for 24 hours. The results are summarised in Table 3 and shown in Fig. 3 and Fig. 4. It appears that the magnitude of the initial offset and the time required for the instrument to stabilise (we refer to this stabilisation time as the "recovery time") depend on the duration of tilt: the longer the instrument remained in the tilted position before recording started, the larger the initial offset and the longer the recovery time. The functional relationship between the magnitude of the initial offset and the duration of tilt is well described by a logarithmic curve (cf. Fig. 4). For instance, a tilt lasting only for 10 minutes may already generate an initial offset of $100 \mu \mathrm{Gal}$ in the data.

The factor that is more important for practical applications is the recovery time as function of the duration of tilt. According to Fig. 4, this relationship is approximately linear. A tilt for 10 minutes causes a recovery time of about 80 minutes; this increases to about 6 hours if the instrument is tilted for 60 minutes. 
Table 3 Initial offset in $\mu \mathrm{Gal}$ and time till readings have stabilised (recovery time) in hours as function of the duration of tilt. In all experiments, the tilt angle is $8^{\circ}$. CG-5 S/N 050300110.

\begin{tabular}{ccc}
\hline duration of tilt $[\mathrm{min}]$ & initial offset $[\mu \mathrm{Gal}]$ & recovery time [hours:minutes] \\
\hline 3 & 0 & 0 \\
4.8 & -25 & $0: 15$ \\
7.6 & -80 & $0: 40$ \\
10.0 & -90 & $1: 21$ \\
13.3 & -106 & $1: 21$ \\
26.8 & -111 & $2: 02$ \\
52.8 & -154 & $3: 20$ \\
74.2 & -185 & $5: 10$ \\
100.7 & -217 & $6: 15$ \\
\hline
\end{tabular}

We found similar relationships between the duration of tilting, initial offset, and recovery time for the other CG-5 meters. These results are shown in Table 4. The functional relationship is not as linear as shown in Fig 4. Sometimes a higher background noise level, perhaps due to uncorrected loading signals, made it difficult to precisely determine the moment in time when the readings stabilised. However, the overall result is unmistakable: initial offset and recovery time increase with the duration of tilt. The recovery time is a factor of $5-10$ longer than the duration of tilt, which is important when designing observation campaigns. This down time differs from what is quoted about the relation between relaxation time and the length of time over which tension of the spring is changed in the Scintrex CG-5 manual (Scintrex Ltd., 2012b).

To answer the second question, Is there an initial offset for any tilt angle or does there exist a critical tilt angle...?, we repeated the first test series for tilt angles varying between $0.9^{\circ}$ and $8.0^{\circ}$. For each tilt angle, the instrument stayed tilted for at least 90 minutes. For 

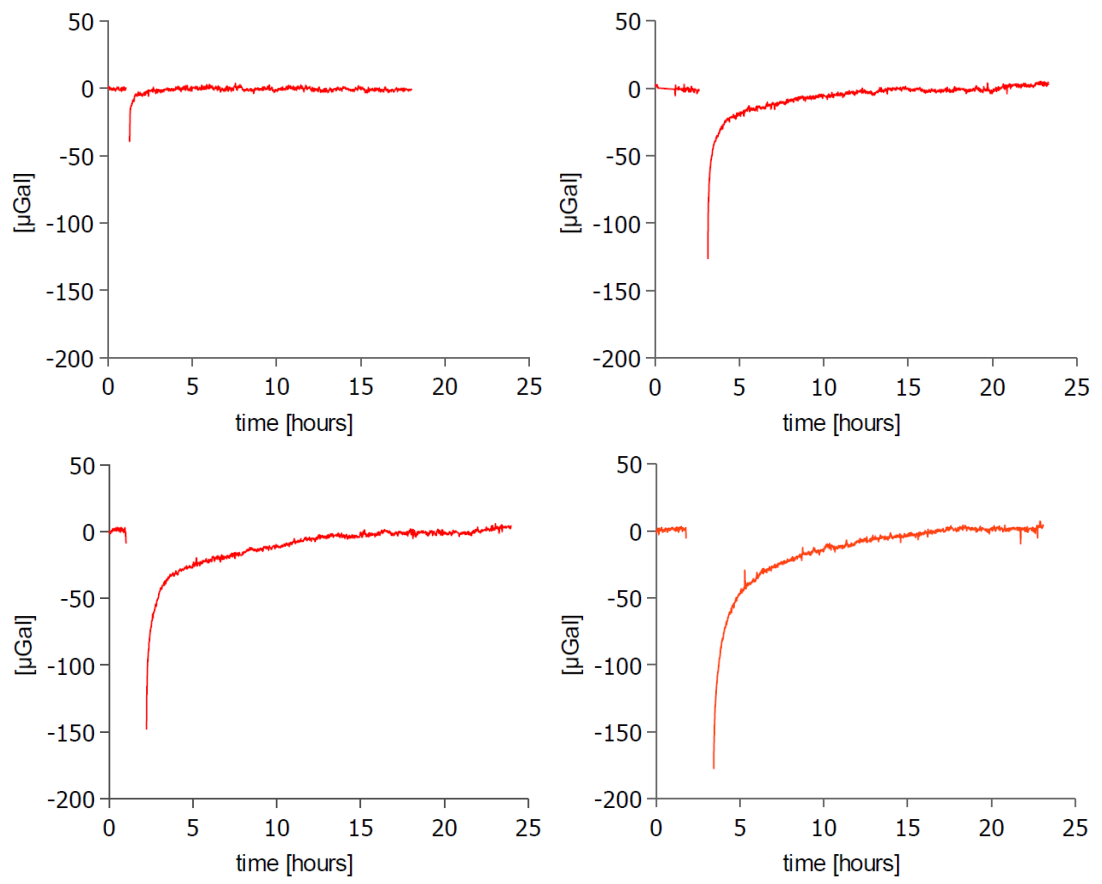

Fig. 3 CG-5 S/N 050300110 readings as function of time. The duration of tilt before readings are recorded is 13 minutes (top left), 27 minutes (top right), 74 minutes (bottom left), and 100 minutes (bottom right), respectively. The tilt angle is always $8^{\circ}$.
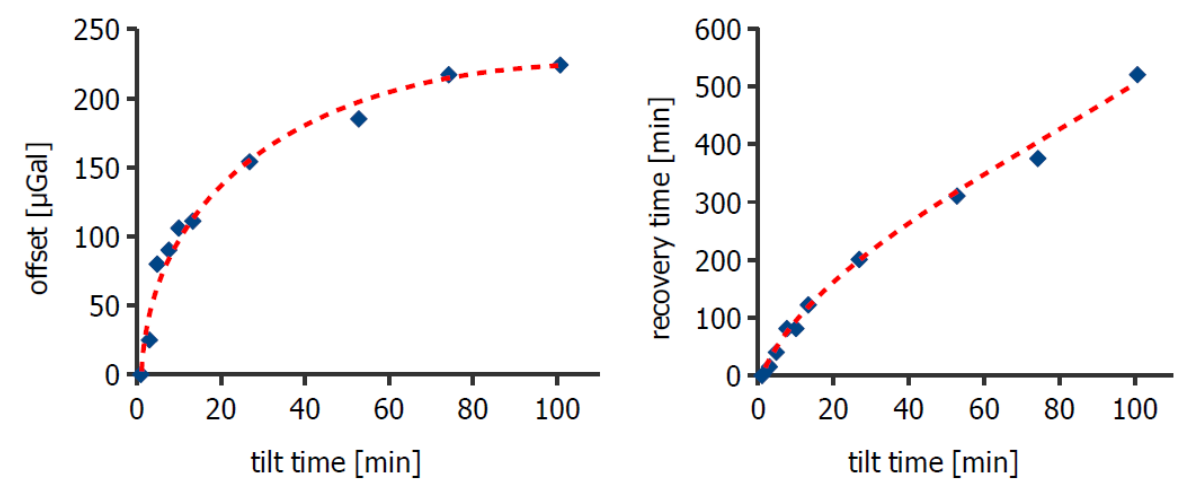

Fig. 4 Initial offset (left) and recovery time (right) as function of the duration of tilt. CG-5 S/N 050300110. Tilt is $8^{\circ}$. 
Table 4 Initial offset in $\mu \mathrm{Gal}$ (first row) and time till readings have stabilised in hours (second row) as function of the the lengths of time in tilted state. In all experiments, the tilt is $8^{\circ}$. "x" means value not available.

\begin{tabular}{crrrr}
\hline duration of tilt & \multicolumn{5}{c}{ CG-5 S/N } \\
\hline min $]$ & 050300110 & 110940807 & 080640191 & 021210010 \\
\hline 10 & -106 & -60 & -30 & -30 \\
30 & 1.4 & 4.0 & 2.0 & 2.0 \\
70 & -154 & -70 & -95 & -100 \\
& 3.3 & 10.0 & 3.5 & 15.0 \\
90 & -217 & -100 & -63 & $\mathrm{x}$ \\
& 6.3 & 12.0 & 4.5 & $\mathrm{x}$ \\
& -220 & -100 & -65 & -110 \\
& 8.7 & 14.0 & 15.0 & 20.0 \\
\hline
\end{tabular}

all instruments we found a critical tilt angle somewhere between $5.2^{\circ}$ and $6.4^{\circ}$. If the tilt is below these critical values, no offsets are observed. If the tilt is above, initial offsets and associated recovery times are much larger than the quoted noise level of the CG-5. We did not attempt to determine the critical tilt angle more accurately for each instrument.

For the three CG-3M meters in Table 2, the situation is somewhat different in the sense that one instrument (CG-3M S/N 0004495) does not show a susceptibility to tilt during the tests, whereas the other two instruments (CG-3M S/N 0004492 and S/N 0004509) were susceptible. For these tilt susceptible instruments, we found initial offsets and recovery times that are smaller than those from the CG-5. However, the offsets and recovery times are still very critical for practical applications. The smaller effects may be due to a lengthening of the spring in the CG-3M meters in the course of time (remember that the CG-3M meters are older than the CG-5 meters) that can accommodate larger tilt angles. Liard et al. (1993) report upon an issue with a CG-3 when the device has been carried on its side between 
sites, something which may happen for instance during commercial aircraft transport. This corresponds to an extreme tilt of $90^{\circ}$. They found that the readings are offset and that the recovery time seems to be proportional to the time the instrument has been on its side. These findings are in agreement with our test results. Liard et al. (1993) do not report on more detailed tests to investigate this problem further.

\section{Summary and recommendations}

We investigated the susceptibility of several Scintrex CG-5 relative gravimeters to tilt, which may easily occur during transits between sites. We found that if the tilt exceeds a critical value of about $6^{\circ}$ that lasts for a minimum of a few minutes, initial observations may be offset by tens of $\mu \mathrm{Gal}$. Thereafter, the offset in the observations decays logarithmically as function of time. Knowledge of this logarithmic decay time is critical for planning field observations as it constrains the time required for the instrument to stabilise to within the noise level. This recovery time easily may take several hours depending on the temporal duration of instrument tilting.

Reviewing the readings at a site for a typical session of 30 minutes as suggested by Scintrex Ltd. (Scintrex Ltd., 2012b) does not provide evidence of an existing offset. We strongly advise users to avoid tilting the CG-5 in excess of $6^{\circ}$ for any prolonged period during transport. The "user sensor check voltage" will indicate compliance with this minimum tilting condition. Voltages very close to zero indicate significant offsets and, therefore, long recovery times at the site. In this case, we recommend that the operator waits a reasonable time before taking a reading. Voltages significantly above zero can be used as an indicator of unbiased readings and of a quality which is in line with the specifications of Scintrex Ltd. In this case, it is sufficient to wait only 15-30 minutes before taking the first reading. 
The predecessor of the CG-5, the CG-3M, is also susceptible to providing erroneous data after sustained tilting. The CG-3M meters we tested show smaller initial offsets and shorter recovery times as compared to the CG-5 meters. Nevertheless, we strongly recommend that users take similar tilt-mitigating precautions when operating the CG-3M in the field.

Acknowledgements We want to thank two anonymous reviewers and the editor, Chris Jekeli, for providing valuable comments which helped us to improve the manuscript.

\section{References}

Christiansen L, Lund S, Andersen OB, Binning PJ, Rosbjerg D, Bauer-Gottwein, P (2011) Measuring gravity change caused by water storage variations: Performance assessment under controlled conditions. Journal of Hydrology 402: 60-70.

Flury J, Peters T, Schmeer M, Timmen L, Wilmes H, Falk R (2007) Precision gravimetry in the new Zugspitze gravity meter calibration system. Harita Dergisi; Özel Sayi 18: 401406.

Jacob T, Bayer R, Chery J, Le Moigne N (2010) Time-lapse microgravity surveys reveal water storage heterogeneity of a karst aquifer. Journal of Geophysical Research 115, B06402, doi:10.1029/2009JB006616.

Lederer M (2009) Accuracy of the relative gravity measurement. Acta Geodyn. Geomater. 6: 383-390.

Liard J, Gagnon, CG, Hearty DB, Salib P, Flint, TR (1993) Evaluation of the Scintrex CG-3 gravity meter. Geological Survey of Canada, open file 2696.

Parseliunas E, Petroskevicius P, Obuchovski R (2008) Analysis of gravimetric observations made by Scintrex CG-5. Selected papers, 7th International Conference on Environmental Engineering, May 22-23, Vilnius, Lithuania, 2008, pages 1422-1428. 
Parseliunas E, Petroskevicius P, Birvydiene R, Obuchovski R (2011) Investigation of the automatic gravimeters CG-5 and analysis of gravimetric measurements. Selected papers, 8 th International Conference on Environmental Engineering, May 19-20, Vilnius, Lithuania, 2011, pages 1416-1423.

Parseliunas E, Petroskevicius P, Obuchovski R, Birvydiene R (2013) An investigation of the automatic relative gravimeters. Solid State Phenomena 199: 261-266.

Scintrex Ltd. (2005) CG-3/3M Autograv automated gravity meter Operator Manual, P.N: 858700 Version 5.00, August 1995. Scintrex Limited, Concord, Ontario.

Scintrex Ltd. (2012) CG-5 Autograv Gravity Meter Brochure, part number 867711, revision 2. Scintrex Limited, Concord, Ontario, Canada

Scintrex Ltd. (2012) CG-5 Scintrex Autograv System Operation Manual, part I \# 867700 Revision 8. Scintrex Limited, Concord, Ontario.

Yushkin VD (2011) Operating experience with CG5 gravimeters. Measurement Techniques 54: 486-489.

Zumberge M, Alnes H, Eiken O, Sasagawa G, Stenvold T (2008) Precision of seafloor gravity and pressure measurements for reservoir monitoring. Geophysics 73 (6): WA133WA141, dpi:10.1190/1.2976777. 